\title{
The effect of dimiristoylphosphatidylethanol on the rotational mobility of $n$-(9-Anthroyloxy) stearic acid in neuronal and model membranes
}

\author{
Young-Sik Park, No-Soo Park, Jun-Bong Seo, Soo-Kyoung Bae, You-Kwang kim, Ki-Soo \\ Seong, Young-Jun Kim, Ju-Won Park, Joung-Moung Shin, Young-Chan Jeon, In-Kyo Chung, \\ Moon-Kyoung Bae, Hye-Ock Jang*, II Yun*
}

School of Dentistry and Research Institute for Oral Biotechnology Pusan National University, Beomeo-ri, Mulgeum-eup, Yangsan, Gyeongsangnam-do, Korea; *Corresponding Author: iyun@pusan.ac.kr, jho9612@pusan.ac.kr

Received 19 August 2010; revised 20 September 2010; accepted 25 September 2010.

\begin{abstract}
The aim of this study was to provide a basis for examining the molecular mechanism for the pharmacological action of ethanol. We studied dimyristoylphosphatidylethanol (DMPEt)'s effects on specific locations of $\boldsymbol{n}$-(9-anthroyloxy) palmitic acid or stearic acid (n-AS) within phospholipids of synaptosomal plasma membrane vesicles isolated from bovine cerebral cortex (SPMV) and liposomes of total lipids (SPMVTL) and phospholipids (SPMVPL) extracted from SPMV. DMPEt increased rotational mobility (increased disordering) of hydrocarbon interior, but it decreased mobility (increased ordering) of membrane interface, in native and model membranes. The degree of rotational mobility in accordance with the carbon atom numbers of phospholipids comprising neuronal membranes was in the order at the 16, 12, 9, 6 and 2 position of aliphatic chain present in phospholipids. The sensitivity of increasing or decreasing effect of rotational mobility of hydrocarbon interior or surface region by DMPEt differed depending on the neuronal and model membranes in the descending order of SPMV, SPMVPL and SPMVTL.
\end{abstract}

Keywords: Ethanol; Phosphatidylethanol; Fluorescence Quenching Technique; Rotational Mobility; Hydrocarbon Interior; Membranes Interface

\section{INTRODUCTION}

The molecular mechanism of action of ethanol in the central nervous system (CNS) has long been a subject of great interest. There are several proposals for the mo- lecular mechanism of action of ethanol, and include lipid [1-6], protein [7-13] and hydration [14-16] theories. However, the precise location of molecular action has continued to be a subject of controversy to the present day.

We have recently shown that a pathway of ethanol metabolism with an unusual phospholipid, dimyristoylphosphatidylethanol (DMPEt) increased the bulk lateral and rotational mobilities, and annular lipid fluidity of neuronal membrane lipid bilayers, and had a greater fluidizing effect on the outer monolayer [17]. We also have shown that DMPEt decreased the thickness of the neuronal membrane lipid bilayer in a dose-dependent manner with a significant decrease in thickness observed at 5 $\mu \mathrm{M}$. There is a good possibility that the effects of ethanol on the CNS result not only from a direct action on neuronal membranes but also from the action of DMPEt [17]. However the fluidity of the membrane outer monolayers may vary at different positions [18,19].

Previous studies have shown that the fluorophores of anthroyloxy derivative locate at a graded series of levels from the surface to the center of the lipid bilayer structure (or a series of anthroyloxy fatty acids indicates that the depth of the group is almost linearly related to the number of carbon atoms between it and carboxyl group) [18-23]. The fluorophores of anthroyloxy derivatives can also be used to differentiate whether the bilayer has a fluidity gradient across it, as the anthrolyoxy group can be positioned at different positions of the stearic acid moiety [18-20,24-27]. These probes have been suggested to measure primarily the dynamic component of membrane fluidity $[18,19,25,28-31]$.

The aim of this research is to provide a basis for studying the molecular mechanism of pharmacological action of DMPEt and ethanol. This study was done 
through investigation of the effect of DMPEt on rotational mobility of the hydrocarbon interior and polar region (membranes interface, surface region) in the native and model membrane which differ in fluidity, and was done through investigation of magnitude of differential sensitivity between native and model membrane to the fluidizing or ordering effect of DMPEt. The study was carried out using 16-(9-anthroyloxy)palmitic acid (16-AP), 12-(9-anthroyloxy)stearic acid (12-AS), 9-(9anthroyloxy) stearic acid (9-AS), 6-(9-anthroyloxy) stearic acid (6-AS) and 2-(9-anthroyloxy)stearic acid (2-AS) those reflecting mobility at the 16, 12, 9, 6 and 2 position of aliphatic chains present in phospholipids of neuronal and model membranes.

\section{MATERIALS AND METHODS}

\subsection{Materials}

The fluorescent anthroyloxy palmitate or stearate probes, 16-AP, 12-AS, 9-AS, 6-AS and 2-AS were purchased from Molecular Probes, Inc. (Junction City, OR, USA). 1, 2-DMPC, phospholipase D, bovine serum albumin (BSA) and other reagents were obtained from Sigma (St. Louis, MO, USA) and were of analytical grade. Phospholipase D was partially purified from Savoy cabbage, using acetone precipitation, according to the method of Davidson and Long [32]. After acetone precipitation the enzyme was suspended in water and lyophilized. The powder obtained was stored at $-20^{\circ} \mathrm{C}$.

\subsection{Synthesis of DMPEt}

DMPEt was synthesized by the procedure of OmodeoSalê et al. [33], which involved the transphosphatidylation of 1, 2-DMPC/ethanol by phospholipase D. The yield was $70-80 \%$, with the DMPEt produced purified by ion-exchange chromatography and characterized by fast atom bombardment-mass spectrometry (FAB-MS).

\subsection{SPMV Preparation}

The SPMV were prepared according to the procedure reported from [34,35]. The specific activities of $\mathrm{Na}$, $\mathrm{K}$-ATPase, acetylcholinesterase and 5'-nucleotidase in the plasma membrane fraction were approximately 4-, 2.5- and 3-times higher than those in crude homogenates. The electron microscopic examination of the prepared SPMV showed very high purity. The vesicles, which were separated according to size, demonstrated homogeneous distribution and no longer showed the presence of intracellular organelles or leakage. The protein concentration was determined by the method of Lowry et al. [36] using BSA as a standard.

\subsection{Liposome Preparation}

Total lipids were extracted from the SPMV as previously described [34]. Cholesterol content of the extracted total lipids was determined according to the Liebermann-Buchard reaction [37]. Phospholipids were quantitated by measuring the amounts of inorganic phosphate [38] after hydrolysis of the phospholipids at $180^{\circ} \mathrm{C}$ in $70 \% \mathrm{HClO}_{4}$ [39]. The SPMV had a high lipid to protein ratio (0.942 $\mathrm{mg}$ total lipids/ $1 \mathrm{mg}$ protein) and a low cholesterol to phospholipid molar ratio. The value was $0.593 \pm 0.011$ (cholesterol $0.208 \pm 0.010$, phospholipids $0.702 \pm 0.025$ ). An average molecular weight of 775 for phospholipids is assumed and the molecular weight of cholesterol is 387 for the calculation. Phospholipids were composed (mol\%) of phosphatidylcholine (41.55 \pm 0.91$)$, phosphatidylethanolamine (36.83 \pm $0.48)$, phosphatidylserine (13.60 \pm 0.26$)$, sphingomyelin $(4.15 \pm 0.16)$, phosphatidylinositol $(2.90 \pm 0.09)$ and lysophosphatidylcholine $(0.97 \pm 0.03)$.

The characteristics of the lipid samples, such as size, lamellarity, radius of curvature, and shape, are strongly dependent on the method used to form the vesicles $[18,40,41]$. As a consequence of the preparation method, the parameters that characterize the lipid phase equilibrium in lipid mixtures are affected by the lipid sample characteristics. Because the size of GUVs is on the same order as the size of cells, GUVs are becoming objects of intense scrutiny in diverse areas that focus on membrane behavior [18,41,42]. Stock solutions of total lipids or phospholipids were made in chloroform. The concentration of the lipid stock solutions was 0.2 $\mathrm{mg} / \mathrm{ml}$. Giant unilamellar vesicles (GUVs: SPMVTL or SPMVPL) with a mean diameter of $45 \mu \mathrm{m}$ were prepared by the method developed [43-45]. To grow the GUVs, a special temperature-controlled chamber, which was previously described $[41,46]$, was used. The experiments were carried out in the same chamber after the vesicle formation, using an inverted microscope (Axiovert35: Zeiss, Thornwood, NY). The following steps were used to prepare the GUVs: 1) $\sim 3 \mu$ of the lipid stock solution was spread on each Pt wire under a stream on $\mathrm{N}_{2}$. To remove the residues of organic solvent, we put the chamber in a liophilizer for $\sim 2$ h. 2) To add the aqueous solvent inside the chamber (Millipore water $17.5 \mathrm{M} \Omega / \mathrm{cm}$ ), the bottom part of the chamber was sealed with a coverslip. The Millipore water was previously heated to the desired temperature $\left(80^{\circ} \mathrm{C}\right.$ for SPMVTL, $60^{\circ} \mathrm{C}$ for SPMVPL), and then sufficient water was added to cover the Pt wires. Just after this step, the Pt wires were connected to a function generator (Hewlett-Packard, Santa Clara, CA), and a low-frequency $\mathrm{AC}$ field (sinusoidal wave function with a frequency of $10 \mathrm{~Hz}$ and an amplitude of $3 \mathrm{~V}$ ) was applied 
for $90 \mathrm{~min}$. After the vesicle formation, the AC field was turned off.

\subsection{Fluorescence Measurements}

The fluorescence measurements were taken using a modified method of earlier study $[18,19,47]$. The SPMV were suspended in PBS to concentration $50 \mu \mathrm{g}$ of protein $/ \mathrm{ml}$. The liposomes (SPMVTL and SPMVPL) were suspended in PBS to give a concentration $0.2 \mathrm{mg}$ of total lipids or total phospholipids/ml. Stock solutions of the 16-AP, 12-AS, 9-AS, 6-AS and 2-AS in methanol (2 $\times 10^{-5} \mathrm{M}$ ) were prepared and kept in a cold and dark place. Aliquots were added to the solutions of the native and model membrane so that the final concentrations of the 16-AP, 12-AS, 9-AS, 6-AS and 2-AS became $4 \times$ $10^{-8} \mathrm{M}$ (in the case of SPMV) or $2 \times 10^{-8} \mathrm{M}$ (in the cases of SPMVTL and SPMVPL) incorporated the probes. The mixture was stirred for $20 \mathrm{~min}$ at room temperature in order to reduce the concentration of DMPEt that might alter the rotational mobility of the SPMV, SPMVTL and SPMVPL. Also, the mixture was bubbled by dry nitrogen for $1 \mathrm{~min}$ with $20 \mathrm{~min}$ intervals in order to eliminate oxygen that might act as a quencher. To ensure complete removal of methanol residue in the mixture, the prepared mixtures were subjected to exhausted stirring for more than $2 \mathrm{hr}$ which have shown the same results as the mixtures stirred for $20 \mathrm{~min}$. Concentrated solution of DMPEt was prepared in PBS and added to the labeled membrane suspension to give the desired concentration of anesthetic. The $\mathrm{pH}$ of the buffered sample was not changed significantly by addition of DMPEt.

Fluorescence measurements were carried out with a Multi Frequency Cross-Correlation Phase and Modulation Fluorometer (ISS K2-003), equipped with a thermostated cell holder and performed at $\mathrm{pH} 7.4\left(37 \pm 0.1^{\circ} \mathrm{C}\right)$. The fluorescent probes, 16-AP, 12-AS, 9-AS, 6-AS and 2-AS, were excited at $360 \mathrm{~nm}$ (4 $\mathrm{nm}$ slit width) and those emissions recorded at $445 \mathrm{~nm}$ (8 nm slit width) through a sharp cut-off filter (Schott KV418). Corrections for light scattering (membrane suspensions without fluorescent probes) and for fluorescence in the ambient medium (quantified by pelleting the membranes after each estimation) were made routinely, and the combined corrections were less than $9 \%$ of the total fluorescence intensity observed for anthroyloxy palmitate or stearateloaded suspensions. The intensity of the components of the fluorescence which were parallel $\left(I_{/ /}\right)$and perpendicular $(I \perp)$ to the direction of the vertically polarized excitation light, was determined by measuring the emitted light through polarizers oriented vertically and horizontally. Polarization $(P)$ was obtained from intensity measurements using $P=\left(I_{/ /}-G I_{\perp}\right) /\left(I_{/ /}+G I_{\perp}\right)$, where $G$ is a grating correction factor for the monochromator's transmission efficiency for vertically and horizontally polarized light. This value is given by the ratio of the fluorescence intensities of the vertical to horizontal components when the exciting light is polarized in the horizontal direction. The polarization was expressed as anisotropy $[r=2 P /(3-P)]$ of 16-AP, 12-AS, 9-AS, 6-AS and 2-AS.

\section{RESULTS}

In the present study, using fluorescence probe technique, we examined the amphiphilic effects of DMPEt on the differential rotational mobility between interface and hydrocarbon interior of SPMV, SPMVTL and SPMVPL. In order to determine effects of the DMPEt on the aforementioned rotational mobility, it was first necessary to demonstrate that the drug did not interact directly with fluorescent probes and thereby quench its fluorescence. Quenching of absorbance-corrected fluorescence intensity by the drug is not observed at all of the concentration levels where DMPEt was tested. Furthermore, if direct quenching of 16-AP, 12-AS, 9-AS, 6-AS and 2-AS by the DMPEt occurred, fluorescence lifetime would decrease. However, the fluorescence lifetime of 16-AP was not changed by the drug in the SPMV. For example, the lifetime of 16-AP in the SPMV was 11.5 $\pm 0.2(\mathrm{n}=5), 11.1 \pm 0.1(\mathrm{n}=5), 11.6 \pm 0.3(\mathrm{n}=5), 11.7 \pm$ $0.1(\mathrm{n}=5)$ and $11.3 \pm 0.2(\mathrm{n}=5) \mathrm{ns}$ at $5,10,15,20$, and 25 $\mu \mathrm{M}$ DMPEt, respectively. Similar results were with 12-AS, 9-AS, 6-AS and 2-AS. Direct quenching of probe fluorescence by the drug used in the present experiments was ruled out. The anisotropy $(r)$ values of 16-AP for hydrocarbon interior of intact SPMV, SPMVTL and SPMVPL were $0.092 \pm 0.002(\mathrm{n}=5), 0.070 \pm 0.001(\mathrm{n}=5)$ and $0.047 \pm 0.001(\mathrm{n}=5)$ at $37^{\circ} \mathrm{C}(\mathrm{pH} 7.4)$ respectively (Table 1). In contrast, the values of 2-AS for interface of intact SPMV, SPMVTL and SPMVPL were $0.126 \pm$ $0.002(\mathrm{n}=5), 0.114 \pm 0.001(\mathrm{n}=5)$ and $0.096 \pm 0.001(\mathrm{n}$ $=5)$ at $37^{\circ} \mathrm{C}(\mathrm{pH} \mathrm{7.4)}$ respectively (Table 1). This means

Table 1. Fluorescence parameters of 16-AP, 12-AS, 9-AS, 6-AS and 2-AS in SPMV.

\begin{tabular}{ccccccc}
\hline Membranes & Parameter & 16-AP & $12-\mathrm{AS}$ & 9 -AS & 6 -AS & 2-AS \\
\hline \multirow{3}{*}{ SPMV } & \multirow{2}{*}{ Anisotropy } & 0.092 & 0.099 & 0.108 & 0.115 & 0.126 \\
& & \pm & \pm & \pm & \pm & \pm \\
& & 0.002 & 0.002 & 0.001 & 0.002 & 0.002 \\
SPMVTL & \multirow{2}{*}{ Anisotropy } & \pm & \pm & \pm & \pm & \pm \\
& & 0.001 & 0.001 & 0.001 & 0.001 & 0.001 \\
& & 0.047 & 0.051 & 0.082 & 0.087 & 0.096 \\
SPMVPL & \multirow{2}{*}{ Anisotropy } & \pm & \pm & \pm & \pm & \pm \\
& & 0.001 & 0.001 & 0.001 & 0.001 & 0.001 \\
\hline
\end{tabular}

Fluorescence measurements were performed at $37^{\circ} \mathrm{C}(\mathrm{pH} 7.4)$. Values represent the mean $\pm \mathrm{SEM}$ of 5 determinations. 
that rotational mobility of hydrocarbon interior is faster than that of membrane interface. The rotational mobility's degrees of SPMV, SPMVTL and SPMVPL differed depending on the phospholipids, cholesterol and phospholipids are co-present and the proteins, cholesterol and phospholipids are co-present in the descending order of the SPMVPL, SPMVTL and SPMV.

\subsection{Ordering Effects of DMPEt on the Rotational Mobility of the Membrane Interface}

The effect of the DMPEt on the anisotropy $(r)$ of the 2-AS in the interface of SPMV, SPMVTL and SPMVPL are shown in Figures 1-3. The DMPEt increased the anisotropy ( $r$ ) of the 2-AS (decreased rotational mobility) in interface of SPMV, SPMVTL and SPMVPL in a concentration-dependent manner. The significant increase in the anisotropy $(r)$ value by DMPEt was observed even at $10 \mu \mathrm{M}$ (Figures 1-3), respectively. The anisotropy $(r)$ values of the 2-AS in interface of SPMV, SPMVTL and SPMVPL were raise by $0.006,0.004$ and 0.006 , respectively, than those in the same region when $25 \mu \mathrm{M}$ DMPEt was added. Variations in the anisotropy $(r)$ values were also noticed by the change in temperature as mentioned earlier. At $37^{\circ} \mathrm{C}(\mathrm{pH} \mathrm{7.4)}$, the anisotropy $(r)$ of the 2-AS in interface of SPMV, SPMVTL and SPMVPL are $0.126 \pm 0.002(n=5), 0.114 \pm 0.001(n=5)$ and $0.096 \pm 0.001(\mathrm{n}=5)$, respectively. On the other hand, at $25^{\circ} \mathrm{C}(\mathrm{pH} \mathrm{7.4)}$, the anisotropy $(r)$ of the $2-\mathrm{AS}$ in interface of SPMV, SPMVTL and SPMVPL are $0.165 \pm$ $0.003(\mathrm{n}=5), 0.157 \pm 0.002(\mathrm{n}=5)$ and $0.142 \pm 0.001(\mathrm{n}$ $=5$ ), respectively. Based on the results obtained at the two different temperatures, the observed effects by the addition of $25 \mu \mathrm{M}$ DMPEt, different values $0.006,0.004$ and 0.006 were comparable to the effect of the temperature changes by approximately $1.8,1.1$ and $1.6^{\circ} \mathrm{C}$, respectively.

\subsection{Disordering Effects of DMPEt on the Rotational Mobility of the Hydrocarbon Interior}

The effect of increasing concentrations of the DMPEt on the anisotropy $(r)$ of the 16-AP, 12-AS, 9-AS and 6-AS in the hydrocarbon interior of SPMV, SPMVTL and SPMVPL is shown in Figures 1-3. The DMPEt decreased the anisotropy $(r)$ of the 16-AP, 12-AS, 9-AS and 6-AS (increased rotational mobility) in a concentration-dependent manner. The significant decreases in the anisotropy $(r)$ values of the 16-AP by the DMPEt in the SPMV, SPMVTL and SPMVPL were observed even at such low concentrations as $10 \mu \mathrm{M}$, respectively (Figures 1-3). The significant decreases in the anisotropy ( $r$ ) val- ues of the 12-AS by the drug in the SPMV, SPMVTL and SPMVPL were observed even at such low concentrations as $10 \mu \mathrm{M}$, respectively (Figures 1-3). The significant decreases in the anisotropy $(r)$ values of the 9-AS by the DMPEt in the SPMV, SPMVTL and SPMVPL were observed even at such low concentrations as 10, 15 and $15 \mu \mathrm{M}$, respectively (Figures 1-3). The DPMEt decreased the anisotropy $(r)$ of the 6-AS in

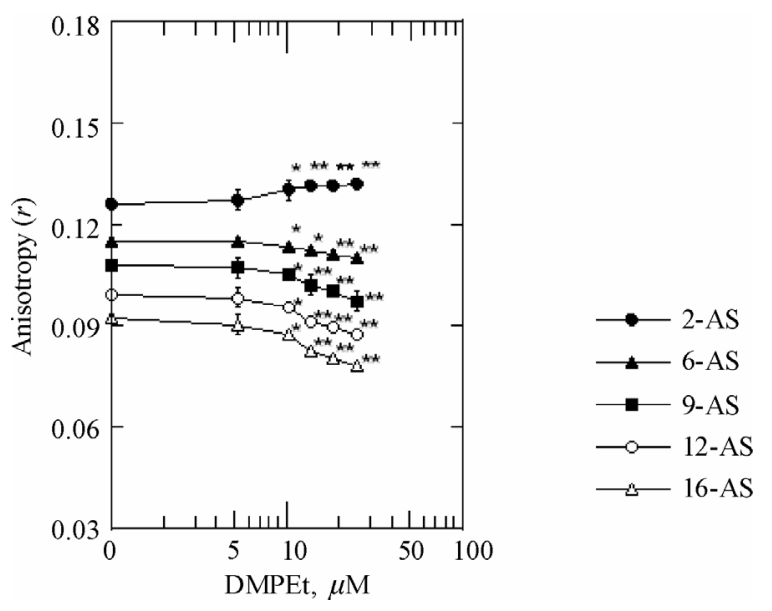

Figure 1. The effect of DMPEt on the anisotropy $(r)$ of the 2-AS, 6-AS, 9-AS, 12-AS and 16-AP in the SPMV. Fluorescence measurements were performed at $37^{\circ} \mathrm{C}(\mathrm{pH} 7.4)$. Each point represents the mean \pm SEM of 5 sample determinations. An asterisk and double asterisks signify $P<0.05$ and $P<0.01$, respectively, compared to control according to Student's $t$-test.

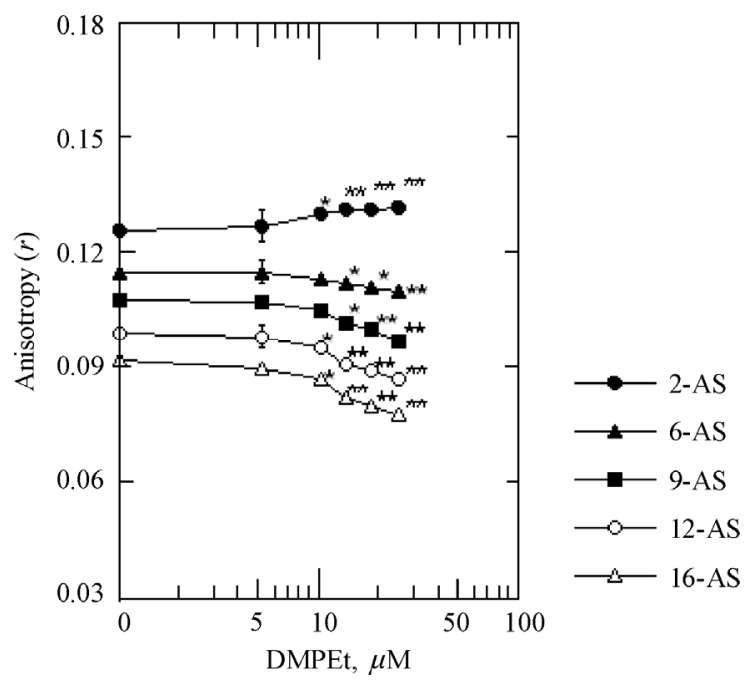

Figure 2. The effect of DMPEt on the anisotropy $(r)$ of the 2-AS, 6-AS, 9-AS, 12-AS and 16-AP in the SPMVTL. Fluorescence measurements were performed at $37^{\circ} \mathrm{C}(\mathrm{pH}$ 7.4). Each point represents the mean \pm SEM of 5 sample determinations. An asterisk and double asterisks signify $P<0.05$ and $P<0.01$, respectively, compared to control according to Student's $t$-test. 


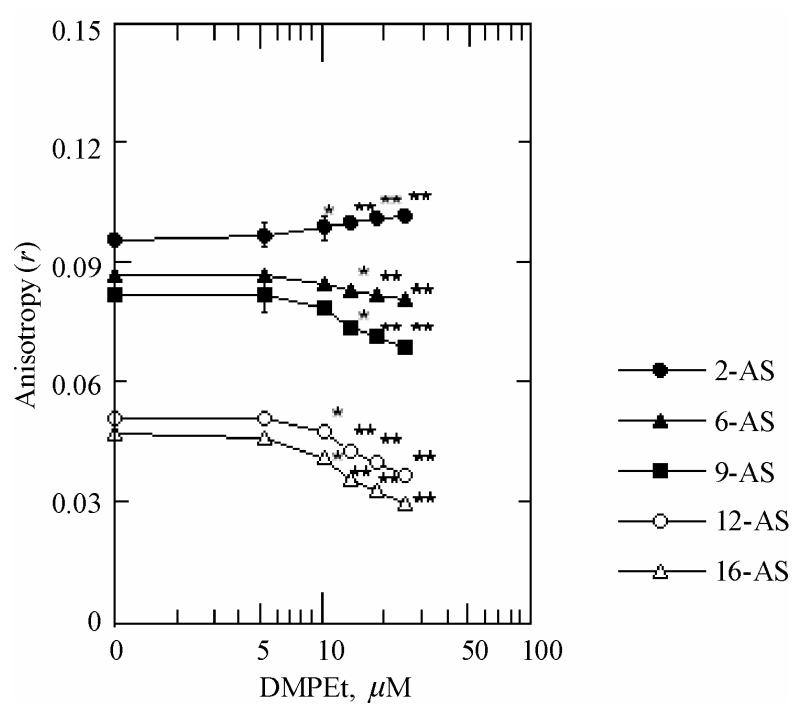

Figure 3. The effect of DMPEt on the anisotropy $(r)$ of the 2-AS, 6-AS, 9-AS, 12-AS and 16-AP in the SPMVPL. Fluorescence measurements were performed at $37^{\circ} \mathrm{C}(\mathrm{pH} 7.4)$. Each point represents the mean \pm SEM of 5 sample determinations. An asterisk and double asterisks signify $P<0.05$ and $P<0.01$, respectively, compared to control according to Student's $t$-test.

a dose-dependent manner and the decrease in anisotropy $(r)$ values in the SPMV, SPMVTL and SPMVPL by the drug was considerable at lower concentration of 10, 15 and $15 \mu \mathrm{M}$, respectively (Figures 1-3). The magnitude of the increased rotational mobility by the DMPEt was in the order at the position of 16, 12, 9 and 6 of aliphatic chains in phospholipids of neuronal and model membrane.

The differences in the anisotropy $(r)$ values of the 16-AP found in hydrocarbon interior of SPMV, SPMVTL and SPMVPL before and after adding $25 \mu \mathrm{M}$ DMPEt was $0.014,0.014$ and 0.017 , respectively. These can be illustrated by comparing effects of temperature on this parameter. The anisotropy $(r)$ of the 16-AP in hydrocarbon interior of SPMV, SPMVTL and SPMVPL are $0.092 \pm 0.002(\mathrm{n}=5), 0.070 \pm 0.001(\mathrm{n}=5)$ and $0.047 \pm$ $0.001(\mathrm{n}=5)$ at $37^{\circ} \mathrm{C}(\mathrm{pH} 7.4)$, respectively. The anisotropy $(r)$ of the 16-AP in hydrocarbon interior of SPMV, SPMVTL and SPMVPL are $0.122 \pm 0.003(\mathrm{n}=5), 0.106$ $\pm 0.001(\mathrm{n}=5)$ and $0.086 \pm 0.001(\mathrm{n}=5)$ at $25^{\circ} \mathrm{C}(\mathrm{pH}$ 7.4), respectively. Thus, the differences in the anisotropy $(r)$ values at the position of 16 in hydrocarbon interior of SPMV, SPMVTL and SPMVPL before and after adding $25 \mu \mathrm{M}$ DMPEt was $0.014,0.014$ and 0.017 , which were as large as those produced by the temperature raises of approximate $5.6,4.7$ and $5.2^{\circ} \mathrm{C}$, respectively.

\section{DISCUSSION}

We paid a special attention to the study by Villalaín and Prieto [21] among several studies [20-24,27] in regard to 2-AS distribution in membranes. The study [21] clearly indicated the distribution region. It was reported that the 2-AS is a peculiar member of the family of probes, as the chromophore is adsorbed on the membrane interface [21]. Membrane interface is difficult to define precisely and it certainly includes the polar region near the phospholipid headgroups, or even the carbonyl groups, which are largely involved in hydrogen bonds. The degree of rotational mobility in according with the carbon numbers of phospholipids comprising of neuronal and model membranes was in the order at the position of $16,12,9,6$, and 2 of aliphatic chains in phospholipids, respectively. Using the membrane interface probe 2-AS, we found that DMPEt decreased the rotational mobility of lipids in the interface of neuronal and model membrane. On the contrary, using the hydrophobic interior probe 16-AP, 12-AS, 9-AS and 6-AS, we found that DMPEt increased rotational mobility of lipids in hydrophobic region of neuronal and model membrane. Furthermore, we found that in terms of increase or decrease of mobility of the neuronal membrane lipid bilayers by DMPEt, the magnitude of effects of DMPEt was greater in neuronal than model membranes.

In a dose-dependent manner, the DMPEt used in the present study lowered the anisotropy $(r)$ values of 16-AP, 12-AS, 9-AS and 6-AS in hydrocarbon interior of SPMV but increased anisotropies of 2-AS in interface of the native and model membrane.

The DPMEt may induce disordering or ordering in their host lipids. Ordering takes place in membrane interface, whereas disordering occurs deep within the acyl chains. Although the DMPEt has strong disordering effects on hydrocarbon interior of native and model membrane, ordering effects of DMPEt on membrane interface are minor $\left(\mathrm{pH} 7.4,37^{\circ} \mathrm{C}\right)$. This is consistent with the findings of Smith et al. [48] and Yun et al. [18]. This has been interpreted in terms of different locations within the bilayer for the charged and neutral forms of the anesthetic; the neutral form is located deeper within the acyl chains and consequently the DMPEt has a lesser effect on the head group $[18,48]$.

Our data on DMPEt suggest that the observed anisotropy $(r)$ values reflect differences in disordering or ordering constraints in hydrocarbon interior and interface of SPMV, SPMTL and SPMVPL. This is due to differences in the intrinsic component and/or the structure in interface and hydrocarbon interior of the native and model membrane. The mechanism of the action(s) of DMPEt on disordering and ordering effects on the neuronal and model membranes is not well-understood. Water associates with the head group region of phospholipids via hydrogen bonding [49]. DMPEt bind (the 
competitive binding of the DMPEt and water) strongly to the phosphate moiety of the phospholipids in membrane interface and weakly to the carbonyl group in competition with water, and effectively establish formation of hydrogen bonds with the carbonyl moiety, which is associated with a significant change in hydration of the DMPEt molecules themselves [50]. Incorporation of DMPEt into the native and model membranes cause alterations of the interface's charge density of the membrane, and a conformational change in phospholipid head groups [50]. At the same time, DMPEt may exert a significant influence on hydration of the lipid bilayer. As a result, such competitive binding decreases rotational mobility and increases hydrophobicity [50]. The interaction of the DMPEt with the hydrocarbon interior will generate rearrangements of the intermolecular hydrogen-bonded network among phospholipid molecules and/or protein molecules that are associated with the liberation of hydrated water molecules on the native and model membranes [50]. The interaction will also change the orientation of the P-N dipole of phospholipid molecules [51]. These changes should cause disordering of the hydrocarbon interior, and thus they could affect the transport of $\mathrm{Na}^{+}$and $\mathrm{K}^{+}$in nerve membranes, leading to anesthetic action.

The sensitivities to the increasing effect of the rotational mobility of the hydrocarbon interior by the DMPEt differed depending on the native and model membranes in the descending order of the SPMV, SPMVPL and SPMVTL. When we take the results of this study and Jang's previous study [17,52], it is without a doubt that the DMPEt increases the rotational mobility of the hydrocarbon interior of the membrane. What could be the reason that the effects on neuronal membranes where phospholipids, cholesterol and proteins are co-present are greater compared to the effect on model membranes where the protein is not co-present? It is presumed because proteins magnify the effect of DMPEt on lipids through protein-lipid interaction. These effects are not solely due to the influence of the DMPEt on lipids, but they are magnified by the interaction between lipids, proteins and water. Water plays a fundamental role in cell membrane structure in that it drives the formation of the lipid bilayer, with a polar surface facing the aqueous environment and a hydrophobic interior containing the fatty acyl chains and transmembrane proteins. In general, the structure and dynamics of proteins are also to a large extent governed by interactions with water [53]. Water penetrates into lipid bilayers at least as far as the glycerol backbone and also deeper between fatty acyl chain packing defects. Water at the proteinlipid interface is an additional factor involved in influencing the lipid bilayer structure. The introduction of small peptides, consisting of three amino acids, can cause a shift of water deeper into the bilayer, indicating increased hydration [54]. Altered hydration may have marked effects on membrane protein/lipid functioning, possibly due to the formation of hydrogen bonds between the interchain water and protein amino acid side chains facing/lipid acyl chains facing into the hydrophobic interior of the membrane. It is possible that the proteins organize the lipid in a way that makes them more susceptible to the drug.

Ethanol increased the lateral and the rotational mobilities of plasma membrane vesicles (CHOK1-PMV) of cultured Chinese hamster ovary K1 cells [5], the plasma membrane vesicles (ATCC-PMV) of cultured hybridoma cells (ATCC T1B 216) [55], plasma membrane vesicles of the cultured mouse myeloma cell line Sp2/0-Ag14 [6] and SPMV [56]. Ethanol had a greater effect on increasing the range of rotational mobility of the outer monolayer compared to the inner monolayer of CHOK1PMV [5], ATCC-PMV [55], Sp2/0-Ag14 [6] and SPMV [56]. Furthermore, as mentioned in the introduction, a pathway for ethanol metabolism, where the product was an unusual phospholipid, PET [57], has been reported. Judging from the results of the present study, as well as those from other studies [17,33,57,58], there is a good possibility that the effects of ethanol on the CNS result not only from a direct action on neuronal membranes, but also from the action of DMPEt.

\section{REFERENCES}

[1] Seeman, P. (1972) The membrane actions of anesthetics and tranquilizers. Pharmacological Reviews, 24, 583-655.

[2] Richards, C.D., Martin, K., Gregory, S., Eightley, C.A., Hesketh, T.R., Smith, G., Warren, G. B. and Metcalfe, J.C. (1978) Degenerate perturbation of protein structure are as the mechanism of anesthetic action. Nature, 276, 775779.

[3] Chin, J.H. and Goldstein, D.B. (1984) Cholesterol blocks the disordering effects of ethanol in biomembranes. Lipids, 19, 929-935.

[4] Incerpi, S., Jefferson, J.R., Wood, W.G., Ball, W.J. and Schroeder, F. (1992) Na pump and plasma membrane structure in L-cell fibroblasts expressing rat liver fatty acid binding protein. Archives of Biochemistry and Biophysics, 298, 35-42.

[5] Yun, I., Yang, M.-S., Kim, I.-S. and Kang, J.-S. (1993) Bulk vs. transbilayer effects of ethanol on the fluidity of the plasma membrane vesicles of cultured Chinese hamster ovary cells. Asia Pacific Journal of Pharmacology, 8, 9-16.

[6] Kang, J.-S., Choi, Ch.-M. and Yun, I. (1996) Effects of ethanol on lateral and rotational mobility of plasma membrane vesicles isolated from cultured mouse myeloma cell line Sp2/0-Ag14. Biochimica et Biophysica Acta, 1281, 157-163.

[7] Halsey, M.J. and Smith, E.B. (1970) Effects of anaes- 
thetics on luminous bacteria. Nature, 227, 1363-1365.

[8] Ueda, I. and Kamaya, H. (1973) Kinetic and thermodynamic aspects of the mechanism of general anesthesia in a model system of firefly luminescence in vitro. Anesthesiology, 38, 425-436.

[9] Franks, N.P. and Lieb, W.R. (1982) Molecular mechanism of general anesthesia. Nature, 300, 487-493.

[10] Franks, N.P. and Lieb, W.R. (1984) Do general anaesthetics act by competitive binding to specific receptors? Nature, 310, 599-601.

[11] Franks, N.P. and Lieb, W.R. (1985) Mapping of general anaesthetic target sites provides a molecular basis for cutoff effects. Nature, 316, 349-351.

[12] Dluzewski, A.R., Halsey, M.J. and Simmonds, A.C. (1983) Membrane interactions with general and local ansesthetics: A review of molecular hypothesis of anesthesia. Molecular Aspects of Medicine, 6, 459-573.

[13] Miller, K.W. (1985) The nature of the site of general anesthesia. International Review of Neurobiology, 27, 161.

[14] Stubbs, C.D. and Rubin, E. (1993) Molecular mechanism of ethanol and anesthetic actions; Lipid and protein-based theories. In: Alling, C., Diamond, I., Leslie, S. W., Sun, G.Y. and Wood, W.G., Eds., Alcohol Cell Membranes and Signal Transduction in Brain, Plenum Press, New York, 1-11.

[15] Griepernau, B., Leis, S., Schneider, M.F., Sikor, M., Steppich, D. and Böckmann, R.A. (2007) 1-Alkanols and membranes: A story of attraction. Biochimica et Biophysica Acta, 1768, 2899-2913.

[16] Klacsová, M., Westh, P. and Balgavỳ, P. (2010) Molecular and component volumes of saturated n-alkanols in DOPC + DOPS bilayers. Chemistry and Physics of Lipids, 163, 498-505.

[17] Jang, H.O., Shin, H.G. and Yun, I. (2004) Effects of dimyristoylphosphatidylethanol on the structural parameters of neuronal membranes. Molecules and Cells, 17, 485-491.

[18] Yun, I., Cho, E.S., Jang, H.O., Kim, U.K., Choi, C.H., Chung, I.K., Kim, I.S. and Wood, W.G. (2002) Amphiphilic effects of local anesthetics on rotational mobility in neuronal and model membranes. Biochimica et Biophysica Acta, 1564, 123-132.

[19] Lee, Y.H., Park, N.S., Kwon, J.D., Park, J.S., Shin, G.B., Lee, C.S., Jung, T.S., Choi, N.J., Yoon, J.H., Ok, J.S., Yoon, U.C., Bae, M.K., Jang, H.O. and Yun, I. (2007) Amphiphilic effects of dibucaine $\mathrm{HCl}$ on rotational mobility of n- (9-anthroyloxy)stearic acid in neuronal and model membranes. Chemistry and Physics of Lipids, 146, $33-42$.

[20] Thulborn, K.R. and Sawyer, W.H. (1978) Properties and the locations of a set of fluorescent probes sensitive to the fluidity gradient of the lipid bilayer. Biochimica et Biophysica Acta, 511, 125-140.

[21] Villalaín, J. and Prieto, M. (1991) Location and interaction of $\mathrm{N}$-(9-anthroyloxy)-stearic acid probes incorporated in phosphatidylcholine vesicles. Chemistry and Physics of Lipids, 59, 9-16.

[22] Abrams, F.S. and London, E. (1993) Extension of the parallax analysis of membrane penetration depth to the polar region of model membranes: Use of fluorescence quenching by a spin-label attached to the phospholipid polar headgroup. Biochemistry, 32, 10826-10831.

[23] Mason, J.T. (1994) Properties of phosphatidylcholine bilayers as revealed by mixed-acyl phospholipid fluorescent probes containing $\mathrm{n}$-(9-anthroyloxy) fatty acids. Biochimica et Biophysica Acta, 1194, 99-108.

[24] Tilley, L., Thulborn, K.R. and Sawyer, W.H. (1979) An assessment of the fluidity gradient of the lipid bilayer as determined by a set of n-(9-anthroyloxy) fatty acids ( $\mathrm{n}=$ 2, 6, 9, 12, 16). The Journal of Biological Chemistry, 254, 2592-2594.

[25] Schacter, D., Cogan, U. and Abbott, R.E. (1982) Asymmetry of lipid dynamics in human erythrocyte membranes studied with permeant fluorophores. Biochemistry, 21, 2146-2150.

[26] Collins, J.M., Dominey, R.N. and Grogan, W.M. (1990) Shape of the fluidity gradient in the plasma membrane of living HeLa cells. Journal of Lipid Research, 31, 261270.

[27] Tricerri, M.A., Garda, H.A. and Brenner, R.R. (1994) Lipid chain order and dynamics at different bilayer depths in liposomes of several phosphatidylcholines studied by differential polarized phase fluorescence. Chemistry and Physics of Lipids, 71, 61-72.

[28] Vincent, M., Foresta, B. de, Gallay J. and Alfsen, A. (1982) Fluorescence anisotropy decays of n-(9-anthroyloxy) fatty acids in dipalmitoylphosphatidylcholine vesicles. Localization of the effects of cholesterol addition. Biochemical and Biophysical Research Communications, 107, 914-921.

[29] Schachter, D. (1984) Fluidity and function of hepatocyte plasma membranes. Hepatology, 4, 140-151.

[30] Brasitus, T.A. and Dudeja, P.K. (1985) Correction of abnormal lipid fluidity and composition of rat ileal microvillus membranes in chronic streptozotocin-induced diabetes by insulin therapy. The Journal of Biological Chemistry, 260, 12405-12409.

[31] Brasitus, T.A., Dudeja, P.K., Eby, B. and Lau, K. (1986) Correction by 1-25-dihydroxycholecalciferol of the abnormal fluidity and lipid composition of enterocyte brush border membranes in vitamin $\mathrm{D}$-deprived rats. The Journal of Biological Chemistry, 261, 16404-16409.

[32] Davidson, F.M. and Long, C. (1958) The structure of the naturally occurring phosphoglycerides. 4 . action of cabbage-leaf phospholipase $\mathrm{D}$ on ovolecithin and related substances. Biochemical Journal, 69, 458-466.

[33] Omodeo-Salê, M.F., Cestaro, B., Mascherpa, A., Monti, D. and Masserini, M. (1989) Enzymatic synthesis and thermotropic behavior of phosphatidylethanol. Chemistry and Physics of Lipids, 50, 135-142.

[34] Yun, I. and Kang, J.-S. (1990) The general lipid composition and aminophospholipid asymmetry of synaptosomal plasma membrane vesicles isolated from bovine cerebral cortex. Molecules and Cells, 1, 15-20.

[35] Yun, I., Kim, Y.-S., Yu, S.-H., Chung, I.-K., Kim, I.-S., Baik, S.-W., Cho, G.-J., Chung, Y.-Z., Kim, S.-H. and Kang, J.-S. (1990) Comparision of several procedures for the preparation of synaptosomal plasma membrane vesicles," Archives of Pharmacal Research, 13, 325-329.

[36] Lowry, O.H., Rosebrough, N.J., Farr, A.L. and Randall, R.J. (1951) Protein measurement with the folin phenol reagent. The Journal of Biological Chemistry, 193, 265275 . 
[37] Huang, T.C., Chen, C.P., Wefler, V. and Raftery, A. (1961) A stable reagent for the Liebermann-Burchard reaction. Application to Rapid Serum Cholesterol Determination. Analytical Chemistry, 33, 1405-1407.

[38] Bartlett, G.R. (1959) Phosphorus assay in column chromatography. The Journal of Biological Chemistry, 234, 466-468.

[39] Madeira, V.M.C. and Antunes-Madeira, M.C. (1976) Lipid composition of biomembranes: A complete analysis of sarcoplasmic reticulum phospholipids. Cienc. Biol. (Coimbra), 2, 265-291.

[40] Lasic, D.D. (1988) The mechanism of vesicle formation. Biochemical Journal, 256, 1-11.

[41] Bagatolli, L.A. and Gratton, E. (1999) Two-photon fluorescence microscopy observation of shape changes at the phase transition in phospholipid giant unilamellar vesicles. Biochemical Journal, 77, 2090-2101.

[42] Menger, F.M. and Keiper, J.S. (1998) Chemistry and physics of giant vesicles as biomembrane models. Current Opinion in Chemical Biology, 2, 726-732.

[43] Angelova, M.L. and Dimitrov, D.S. (1986) Liposome electroformation. Faraday discussions of the Chemical Society, 81, 303-311.

[44] Dimitrov, D.S. and Angelova, M.L. (1987) Lipid swelling and liposome formation on solid surfaces in external electric fields. Progress in Colloid and Polymer Science, 73, 48-56.

[45] Angelova, M.L., Soléau, S., Meléard, P.H., Faucon, J.F. and Bothorel, P. (1992) Preparation of giant vesicles by external AC fields, kinetics and application. Progress in Colloid and Polymer Science, 89, 127-131.

[46] Bagatolli, L.A. and Gratton, E. (2000) Two photon fluorescence microscopy of coexisting lipid domains in giant unilamellar vesicles of binary phospholipid mixtures. Biochemical Journal, 78, 290-305.

[47] Molitoris, B.A. and Hoilien, C. (1987) Static and dynamic components of renal cortical brush border and basolateral membrane fluidity: Role of cholesterol. Journal of Membrane Biology, 99, 165-172.

[48] Smith, I.C., Auger, M. and Jarrell, H.C. (1991) Molecular details of anesthetic-lipid interaction. Annals of the New York Academy of Sciences, 625, 668-684.

[49] Boggs, J.M. (1987) Lipid intermolecular hydrogen bonding: Influence on structural organization and membrane function. Biochimica et Biophysica Acta, 906, 353404.

[50] Shibata, A., Ikawa, K. and Terada, H. (1995) Site of action of the local anesthetic tetracaine in a phosphatidylcholine bilayer with incorporated cardiolipin. Biochemical Journal, 69, 470-477.

[51] Scherer, P.G. and Seelig, J. (1989) Electric charge effects on phospholipid headgroups. Phosphatidylcholine in mixtures with cationic and anionic amphiphiles. Biochemistry, 28, 7720-7728.

[52] Jang, H.O., Huh, M.H., Lee, S.W., Lee, Y.H., Lee, J.H., Seo, J.B., Koo, K.I., Jin, S.D., Jeong, J.H., Lim, J.S., Bae, M.K. and Yun, I. (2005) The effect of dimyristoylphosphatidylethanol on the lateral and rotational mobilities of liposome lipid bilayers. Archives of Pharmacal Research, 28, 839-847.

[53] Teeter, M.M. (1991) Water-protein interactions: Theory and experiment. Annual Review of Biophysics and Biophysical Chemistry, 20, 577-600.

[54] Jacobs, R.E. and White, S.H. (1989) The nature of the hydrophobic binding of small peptides at the bilayer interface: Implications for the insertion of transbilayer helices. Biochemistry, 28, 3421-3437.

[55] Yun, I., Lee, S.H. and Kang, J.S. (1994) The effect of ethanol on lateral and rotational mobility of plasma membrane vesicles isolated from cultured mar 18.5 hybridoma cells. Journal of Membrane Biology, 138, 221227.

[56] Bae, M.K., Jeong, D.K., Park, N.S., Lee, C.H., Cho, B.H., Jang, H.O. and Yun, I. (2005) The effect of ethanol on the physical properties of neuronal membranes. Molecules and Cells, 19, 356-364.

[57] Alling, C., Gustavsson, L., Mansson, J.E., Benthin, G. and Anggard, E. (1984) Phosphatidylethanol formation in rat organs after ethanol treatment. Biochimica et Biophysica Acta, 793, 119-122.

[58] Omodeo-Sale, F., Lindi, C., Palestini, P. and Masserini, M. (1991) Role of phosphatidylethanol in membranes. effects on membrane fluidity, tolerance to ethanol, and activity of membrane-bound enzymes. Biochemistry, 30, 2477-2482. 